\title{
Risco Ergonômico na Atividade Envolvendo Transporte Manual de Cargas no Canteiro de Obras
}

\author{
Ergonomic Risk in Activity Involving Manual Load Transport on the Construction Site
}

José Guilherme da Silveira Vasconcelos Ferreira*, Junio da Silva Amorim, Luane da Silva Lavinas, Barbara Braga Barbosa

Como citar esse artigo. Ferreira, JGSV; Amorim, JS; Lavinas, LS; Barbosa, BB; Risco Ergonômico na Atividade Envolvendo Transporte Manual de Cargas no Canteiro de Obras. Revista Teccen. 2019 Jul/Dez; 12 (2): 39-44.

\begin{abstract}
Resumo
Adaptar a forma de trabalhar ao homem ganhou destaque nas últimas décadas e se tornou uma ciência chamada ergonomia, que visa o conforto e a segurança do trabalhador em suas atividades psicofisiológicas, estudando os possíveis riscos que o trabalhador pode sofrer, evitando assim doenças e acidentes de trabalho. Esses riscos ergonômicos na construção civil podem vir desde lesões na pele, que podem ser causadas pela exposição demasiada ao sol, até lesões na coluna vertebral, produto do excesso de carregamento de peso, por exemplo. O presente artigo expõe uma revisão bibliográfica sobre o excesso do manuseio de cargas em canteiros de obras, baseando-se nas medidas e normas regulamentadoras adotadas pelo Ministério do Trabalho. Essa revisão discute tais aspectos e também relata as consequências do transporte incorreto de cargas e as atitudes que devem ser tomadas nesses casos. Esse estudo é elaborado por meio de documentação indireta; um levantamento de dados de outros artigos, monografias e fontes estatísticas. Conclui-se que grande parte dos problemas que o trabalhador sofre nos canteiros de obra está ligada ao carregamento de peso, e, por isso, é preciso a tomada de mudanças e regras, para que o limite da capacidade humana não seja extrapolado.

Palavras-Chave: transporte de carga, construção civil, ergonomia
\end{abstract}

\begin{abstract}
In the latest decades, to adapt the way of working to men has gained notoriety and it became a science called ergonomics, which seeks comfort and worker safety in their psychophysiological activities, studying the possible risks that the employee may suffer, thus preventing diseases and accidents job. These ergonomic hazards in the civil engineering can come from skin lesions, which may be caused by overexposure to the sun, to spinal cord injuries, affected by lifting and hand transporting excess of materials, for example. This article presents a review on the lifting and hand transporting excess of materials at construction sites, based on the measures and official safety norms approved by the Ministry of Labor and Employment. This review discusses these aspects and also reports the consequences of incorrect transporting charges and attitudes that must be taken in such cases. This study was directed by indirect documentation; a data collection of other articles, monographs and statistical sources. It was concluded that the majority of the problems construction site's workers have is connected to weight loading, and, therefore, it is required to make changes and rules, so that the limit of the human capacity be not extrapolated.

Keywords: material transporting, civil engineering, ergonomic
\end{abstract}

\section{Introdução}

A engenharia civil cresceu intensamente à medida que o mundo evoluiu tecnologicamente, entretanto, a saúde mental e física do trabalhador não ganhou a devida atenção. Problemas fisiológicos e psicológicos apareceram e interferiram na qualidade e no desempenho produtivo do trabalhador nos canteiros de obras, onde se faz frequente a realização de esforço físico intenso. Junto a isso, à rotina carregada e à submissão a posturas inadequadas, o homem também tem que lidar com o transporte manual pesado de cargas. Na construção civil, é constante a ocorrência dessas atividades; são utilizados, por exemplo, sacos de cimento, tábuas, blocos, tijolos e vergalhões (Araújo \& Meira, n.d.).

Assim, se estabelece imprescindível uma avaliação ergonômica que dite as normas a serem

Afiliação dos autores: Universidade de Vassouras - UV, Vassouras, RJ, Brasil

* Email para correspondência: jgsvf6@gmail.com 
seguidas nesse meio de produção. Diante disso, a ergonomia se torna necessária na engenharia civil, pois é por meio dela que os problemas encontrados em campo podem ser solucionados, tornando-se indispensável a inserção de especialistas nessa área - comprometidos com a saúde e segurança do trabalho -, e havendo assim o treinamento do grupo de trabalhadores e a conscientização acerca dos riscos associados aos seus serviços e do uso dos equipamentos de proteção individual (EPI) (Borba \& Soares, 2013).

Para Merino (1996), o trabalhador obtém sérios problemas ao transportar cargas excessivas, e um dos principais é a dor lombar, que pode ocasionar casos crônicos e agudos na saúde do homem. Bankoff, Villarta \& Freire (1994) afirmam que o ritmo cardíaco e a pressão sanguínea também podem ser afetados, adicionando ainda outros problemas, como hemorragia cerebral, hérnia abdominal e ptose.

Em vista disso, a ergonomia se faz presente nessa área por meio de recomendações, de modo que estas possam auxiliar e prevenir o aparecimento de problemas como esses apresentados. Dentre estas recomendações, apresentam-se a mecanização da atividade física, as pausas na jornada de trabalho e a utilização dos equipamentos de proteção individual, evidenciando os aspectos organizacionais da empresa (Melo, 2003).

$\mathrm{O}$ presente trabalho tem como objetivo realizar uma abordagem aprofundada acerca das atividades envolvendo manuseio de material nos canteiros de obras, utilizando como referência artigos, como publicações em revistas e estudos de casos, e as normas regulamentadoras, propostas pelo Ministério do Trabalho. Deste modo, pretende-se levantar dados suficientes na literatura, abordando a inserção da Ergonomia na Engenharia Civil e seus recursos, e mostrar a extrema importância e necessidade de cuidado e atenção com esse tema.

\section{Metodologia}

O presente estudo se constitui de uma revisão literária acerca dos riscos ergonômicos no transporte inadequado e excessivo de cargas pelo trabalhador na construção civil, causador de inúmeras e sérias complicações psicológicas e fisiológicas a saúde do homem. A pesquisa é concretizada a partir de artigos científicos, publicações em revistas, trabalhos publicados e apresentados em eventos ou simpósios, estudos de casos em canteiros de obras, monografias e livros. Com base nas normas regulamentadoras de ergonomia, que são propostas até hoje - destacando-se a NR-17 -, este artigo conta com fontes estatísticas, com o intuito de preencher as lacunas do conhecimento e possibilitar a discussão dos dados apresentados.

\section{O Transporte manual de cargas}

De acordo com a norma regulamentadora 17 (NR17), transporte manual de cargas é aquele que o peso do material é suportado inteiramente por apenas um trabalhador, envolvendo o levantamento e a disposição da carga. E transporte manual regular de cargas é aquele realizado continuamente ou não (Brasil, 1978).

Nos canteiros de obras, ocorre muito comumente a realização de atividades envolvendo o levantamento e transporte de cargas, como sacos de cimento, cilindros, chapas de compensado, tábuas, barrotes, blocos, tijolos, vergalhões, etc. (Araújo \& Meira, n.d.). Para Melo (2003), a profissão exercida pelo trabalhador está relacionada fortemente com a manifestação de problemas de saúde. Dessa forma, os postos de serviço da construção civil que exigem uma grande sobrecarga física, realizada com uma postura inadequada, expõem o trabalhador a dores, geralmente na zona lombar do corpo.

Quando essas atividades são realizadas de forma esporádica e sem os devidos cuidados, Bankoff et al. (1994) afirma que os problemas mais constantes são as hemorragias cerebrais, em pacientes com arterioesclerose - endurecimento das artérias; a hérnia abdominal e a ptose - queda ou localização anormalmente baixa de um órgão -, em pessoas com variação de pressão repentina.

Enquanto para Couto (1991 apud Merino, 1996), a repetição de movimentos em conjunto com uma força são fatores que influenciam diretamente no desenvolvimento de LER's (lesões por esforços repetitivos). Estas lesões e/ou distúrbios, segundo D. S. Kassada, Lopes \& D. A. Kassada (2011) apresentam alguns sintomas em comum, dos quais nem sempre são específicos, tais como formigamento, dor, calor, fraqueza muscular, inflamações em tendões, ligamentos e bolsas sinoviais.

Quando o trabalho é frequentemente excessivo, de acordo com Moura (1978), o que ocorre, principalmente em jovens, são a tensão e o esforço constante nas articulações, ligamentos, músculos e ossos. Com isso, deformações podem ser causadas, como escolioses, cifoses vertebrais e deformação do arco do pé, assim como inflamações e dores dos músculos e bolsas articulares, como miosites e bursites.

Para Grandjean (1982), o transporte de cargas pode ser fatal, pois algumas lesões são irreversíveis, como os problemas na coluna, caracterizados como os principais riscos nessa atividade, ocasionando dificuldade na mobilidade e redução na energia dos trabalhadores. Assim como Grandjean, Mendes (1995) afirma que, no mundo todo, as economias dos países têm sofrido prejuízos devido a esses problemas lombares causados nos funcionários, indicando altos níveis de 
abstenção nas empresas e gastos previdenciários.

No Brasil, os dados não são diferentes. Para Mercúrio (1978), 80\% dos trabalhadores têm ou terão problemas na coluna, o que representa a segunda maior razão dos afastamentos no trabalho. Nogueira (1987) afirma que $70 \%$ dos problemas apresentados no país pelos funcionários que manuseiam cargas pesadas são problemas lombares.

\section{Legislação}

De acordo com a Consolidação das Leis Trabalhistas (CLT), no seu Art. 198, seção XIV, referente à presença de fadiga, o peso máximo que um trabalhador pode transportar em sua atividade laboral é estimado em 60 quilos (kg) (Brasil, 1943).

A legislação trabalhista é constituída por um conteúdo detalhado a respeito da segurança e da saúde do trabalhador no ambiente de trabalho, organizado em Normas Regulamentadoras (NR), com diversos temas e questões, que visam esclarecer as ações na área de saúde do colaborador (Servilha, Leal \& Hidaka, 2010).

Dessa forma, serão apresentadas as normas regulamentadoras que discorrem sobre as práticas no trabalho operacional de transporte manual de cargas, impostas pelo Ministério do Trabalho.

\section{NR-5 Comissão Interna de Prevenção de Acidentes (CIPA)}

Com a NR-5, objetiva-se preservar a vida e promover a saúde do trabalhador, por meio da composição de uma comissão interna, chamada Comissão Interna de Prevenção de Acidentes (CIPA) (Brasil, 1978).

A CIPA tem a incumbência de principalmente identificar os riscos do processo de trabalho e elaborar uma ação preventiva na resolução desses problemas, por meio do controle e da implementação de medidas necessárias nos locais de trabalho. De acordo com um calendário preestabelecido, a CIPA deve ser reunida mensalmente para tratar de assuntos relacionados à saúde e à segurança do trabalhador, realizando a avaliação do cumprimento das metas propostas no plano da empresa e a divulgação de informações importantes aos trabalhadores (Brasil, 1978).

\section{NR-9 Programa de Prevenção de Riscos Ambientais (PPRA)}

A NR-9 determina a obrigatoriedade da implementação do Programa de Prevenção de Riscos Ambientais (PPRA), buscando o reconhecimento e o controle de riscos ambientais que venham existir no ambiente de trabalho. Levando em consideração a preservação do meio ambiente e da saúde do homem, o PPRA deve identificar antecipadamente e avaliar um possível risco no trabalho, implantar medidas de controle, e, por fim, monitorar e registrar a exposição do trabalhador a esse risco (Brasil, 1978).

\section{NR-11 Transporte, movimentação, armazenagem e manuseio de materiais}

O transporte, a movimentação, a armazenagem e o manuseio de materiais também são regulamentados para a segurança do trabalhador, na NR-11. Nela, ditamse as regras e as medidas necessárias para tais funções; e definem-se os cálculos corretos que garantem a conservação das condições de trabalho (Brasil, 1978).

Para o transporte manual de sacos, por exemplo, fica estabelecida a distância máxima de 60 metros por saco; para a carga e descarga de sacos, deve haver o auxílio de um ajudante; para o empilhamento de cargas, aconselha-se o uso de máquinas como as esteirasrolantes; e, por fim, na existência de pisos molhados ou escorregadios, o transporte deve ser evitado (Brasil, 1978).

\section{NR-17 Ergonomia}

Com o propósito de gerar máximo conforto, segurança e desempenho aos trabalhadores, a NR17 estabelece meios que apropriem adequadamente as condições de trabalho à saúde psicofisiológica do trabalhador, determinando assim a função da Ergonomia na vida do homem (Brasil, 1978).

O levantamento e o transporte de materiais, os equipamentos, as condições ambientais e a organização do trabalho fazem parte dos aspectos tratados nessa NR. E, para esses aspectos serem tratados, deve ser realizada uma avaliação ergonômica do trabalho, visando assim ao principal objetivo proposto da norma (Brasil, 1978).

Incluso à NR-17, designa-se transporte manual de cargas no qual um só trabalhador suporta totalmente o peso da carga, desde o levantamento até a disposição da carga; proíbe-se o transporte de cargas cujo peso seja capaz de afetar a saúde do trabalhador; obriga-se a realização de treinamentodo trabalhador na sua função, a fim de prevenir acidentes e proteger sua saúde; obrigase a utilização de meios técnicos adequados para limitar ou facilitar a função do empregado; e admite-se para mulheres e jovens o peso máximo de cargas inferior àquele atribuído aos homens (Brasil, 1978).

A NR-17 também disserta sobre as atividades que exigem muita sobrecarga muscular do corpo humano. Para esses casos, deve-se atentar para a saúde do trabalhador, incluindo pausas para o descanso. E, no retorno ao trabalho, após 15 dias ou mais de afastamento, 
a produção deve ser de maneira gradativa ao nível de produção vigente no período anterior ao afastamento (Brasil, 1978).

\section{Resultados e Discussão}

Segundo Waters, Putz-Anderson, Garg, \& Fine (1993, apud. Merino, 1996), os pesquisadores indicam 23 quilos $(\mathrm{kg})$ como o limite de peso recomendado, estabelecido pelo National Institute for Ocupational Safetyand Health (NIOSH), afirmando que mais de $75 \%$ das mulheres e mais de $90 \%$ dos homens podem suportar adequadamente este peso. $\mathrm{O}$ valor de $23 \mathrm{~kg}$ é correspondente ao peso limite ideal, o qual pode ser transportado sem agravos ao corpo humano. Esse método apresenta grande utilidade e fácil aplicação, que contribui para o controle e organização de uma empresa.

Conforme esse critério, o levantamento de 60 kg, estimado pela CLT em seu Art. 198 (Brasil, 1943), ultrapassa o dobro do transporte de carga permitido, causando sérios problemas aos trabalhadores, como lombalgia e hérnia de disco. Dessa forma, o Ministério do Trabalho dispõe deste método no Manual de Aplicação da NR 17 para utilização, adotando o limite de peso de $23 \mathrm{~kg}$ para as atividades envolvendo transporte manual de cargas (Ministério do Trabalho e Emprego, 2012).

\section{Possíveis causas}

A movimentação de cargas pesadas exige do trabalhador enorme esforço físico e, devido sua intensidade, pode ocasionar diversas lesões (Couto, 1995 apud. Melo, 2003). Para Merino (1996), há algumas características no ambiente de trabalho que podem ser as causadoras das lesões. São elas:

- A ausência de máquinas para o manuseio de cargas;

- Funcionários fisicamente incapacitados, o transporte de cargas maiores que os limites de peso suportáveis e a jornada de trabalho exaustiva;

- Os modos inadequados de manusear as cargas;

- A individualização do trabalho;

- A insuficiência de equipamentos de proteção, tanto individual quanto coletiva;

- A falta de higienização, organização e cuidado com o ambiente de trabalho, que podem ser vistos como insegurança do local.

\section{Recomendações}

Em vista de atividades como o transporte manual de cargas, que trazem riscos comprometedores à saúde do trabalhador, D. S. Kassada et al. (2011) afirma que se torna necessária a adequação das condições de trabalho do funcionário quanto à praticidade e ao conforto físico e psíquico do homem. Isso pode ocorrer de diversas maneiras, como a modernização de equipamentos e máquinas, o uso de ferramentas adequadas, a alteração na jornada de trabalho e a melhoria nas condições do local de trabalho.

Ao desempenhar as atividades de levantamento e transporte manual de cargas, os trabalhadores devem estar cientes de algumas indicações. De acordo com a FUNDACENTRO (1991), eles devem se atentar: ao tamanho, às dimensões, e ao peso da carga, para analisar a forma mais segura de levantá-la; à existência de pontas ou rebarbas, para não ocorrer algum acidente; à necessidade de utilizar os equipamentos de proteção individual, como luvas e botas; e à distância e ao caminho a serem percorridos, devendo este estar desimpedido, limpo e não escorregadio.

Além desses cuidados, ao executar o manuseio de cargas, o homem pode seguir algumas instruções, que realizarão uma pressão uniforme no disco intervertebral do funcionário, evitando o aparecimento de diversos problemas à sua coluna; são elas:

\begin{abstract}
Posicionar-se junto à carga, mantendo os pés afastados, com um pé mais à frente que o outro, para aumentar sua base de sustentação; abaixar-se dobrando os joelhos e mantendo a cabeça e as costas em linha reta; segurar firmemente a carga, usando a palma das mãos e todos os dedos; levantar-se usando somente o esforço das pernas, e mantendo os braços estendidos; aproximar bem a carga do corpo; e manter a carga centralizada em relação às pernas durante o percurso (Araújo \& Meira, n.d.).
\end{abstract}

D. S. Kassada et al. (2011) observa algumas práticas de resolução ou redução dos problemas advindos dessas atividades, causados pela não aplicação da ergonomia. Aautora determina a melhoria da organização do sistema de trabalho da empresa, propondo cobranças de atitudes adequadas dos colaboradores, assim como, motiva a prática da orientação do trabalhador acerca do manuseio correto de cargas, como o revezamento de funcionários ou o rodízio de atividades, evitando assim o excesso de atividades repetitivas. Para isso, as organizações devem contratar profissionais com alto nível de instrução para realizar as devidas análises dos riscos ergonômicos nessa área.

A NR-17 da Ergonomia estabelece parâmetros de adaptação das condições de trabalho às características psicofisiológicas dos trabalhadores, visando à segurança, ao bom desempenho e ao conforto do homem (Brasil, 1978). Baseando-se nessa norma regulamentadora, Medeiros (2013), Melo (2003) e Merino (1996) apresentam algumas recomendações quanto à solução dos problemas expostos no presente artigo, demonstrando assim que a ciência da Ergonomia 
desempenha uma função essencial no estudo dessas atividades.

- Mecanização: nas atividades onde o esforço físico do trabalhador é frequentemente intenso ou quando a segurança do trabalho é comprometida, recomenda-se a inserção de meios mecânicos para o devido fim. Com isso, promove-se a facilitação do trabalho do homem, por meio de máquinas, como os manipuladores, balancins, esteiras transportadoras, mesas rotativas, etc.;

- Divisão da carga: se a carga for muito pesada ou de grandes dimensões, é necessário transportá-la por mais de um funcionário, dividindo assim o esforço;

- Pausas: numa jornada de trabalho intensa, o homem necessita de uma pausa, para que haja a sua recuperação física e mental. Conforme a Consolidação das Leis do Trabalho (CLT), as pausas são períodos de tempo conferidos ao funcionário que trabalha em jornadas ininterruptas de mais de seis horas e devem ser usadas para as refeições e o repouso. Deve ser feita uma programação dessas pausas, satisfazendo os períodos entre o início do trabalho, refeição e término da jornada;

- Ginástica laboral: muitos músculos estão submetidos a cargas pesadas nas atividades dos canteiros de obras. Assim, a ginástica laboral se torna necessária, pois contribui na melhoria da coordenação motora, do equilíbrio e da flexibilidade, aliviando algumas musculaturas muito utilizadas e trabalhando as que são pouco solicitadas;

- Equipamentos de proteção individual (EPI): de acordo com a Norma Regulamentadora 6 (NR-6), o uso dos EPI auxilia na redução dos efeitos nocivos do campo de trabalho do funcionário e protege o operário na ocorrência de algum acidente;

- Alimentação: sabe-se que o transporte manual de cargas consome muita energia dos trabalhadores; quanto mais cargas transportadas, maior a energia utilizada. Com isso, uma alimentação adequada deve ser feita, mediada por profissionais qualificados, para que os trabalhadores obtenham a reposição necessária de energia;

- Treinamento: é fundamental a realização de treinamentos frequentes no ambiente de trabalho, a fim de orientar os funcionários acerca da prática correta de suas atividades, eliminando os movimentos ou posturas críticas, por exemplo. Nesses treinamentos, faz-se necessária a abordagem dos perigos ao transportar cargas, do cuidado com o corpo humano e sua vulnerabilidade, envolvendo os assuntos relacionados às posturas adequadas, a uma dieta equilibrada e ao repouso do trabalhador.

\section{Considerações finais}

De acordo com o que foi estudado, tendo como base jurídica as normas regulamentadoras impostas pelo Ministério do Trabalho, pode-se afirmar que existe a necessidade de atenção e cuidado com a saúde do trabalhador nas atividades envolvendo transporte manual de cargas nos canteiros de obras. O manuseio de cargas pode ser caracterizado principalmente pelo deslocamento contínuo e a sua repetitividade, ocasionando diversos agravos ao bem-estar do trabalhador.

Dentre esses agravos, a região lombar do corpo humano é a mais afetada, pois é nela que o esforço ocorre mais intensamente, acarretando uma série de lesões e doenças ao funcionário. Por ignorância ou desleixo quanto à aplicação dos procedimentos corretos nessas atividades, o trabalhador fica à deriva de sérios danos a sua saúde, como escolioses, hérnias abdominais, bursites e até mesmo hemorragias cerebrais.

Perante as Normas Regulamentadoras, é possível se conscientizar acerca dos direitos do trabalhador, pois são elas que determinam as condições mínimas e legais que sustentam os riscos ergonômicos no levantamento de materiais nos canteiros de obras. Tomando 23 quilos $(\mathrm{kg})$ como o limite máximo de peso que uma pessoa pode carregar, baseando-se no método de NIOSH, constata-se que os limites humanos são suprimidos, o que, por fim, ocasiona em malefícios, não somente para a saúde humana, mas também para a produtividade da empresa.

Com isso, tornam-se indispensáveis a noção e a compreensão quanto à adaptação das condições de trabalho do homem no transporte de cargas, trazendolhe o conforto psicofisiológico e, posteriormente, a melhoria e eficiência no seu rendimento operacional. Destaca-se assim, a necessidade de visar à recuperação da saúde do funcionário, extinguindo ou ao menos reduzindo a aparição das doenças ocupacionais. Afinal, tais avanços podem proporcionar grandes resultados de médio a longo prazo à empresa.

Desse modo, conclui-se ser imprescindíveis algumas mudanças nos sistemas de produção das empresas, como a mecanização de algumas funções, a modernização de equipamentos e máquinas e os seus usos adequados. Assim como se pontua de extrema importância a organização do trabalho operacional, sugerindo a divisão das cargas transportadas e a realização de treinamentos e de ginástica laboral, a fim de preparar o trabalhador fisicamente e o habilitar corretamente à realização dessas tarefas. Da mesma forma, estipular períodos de pausa na carga horária do funcionário, garantindo a sua boa alimentação, para que, assim, ocorra a promoção de um ambiente melhor de trabalho.

\section{Referências}

Araújo, N. M. C., \& Meira, G. R. (1996). Riscos ergonômicos em canteiros 
de obras de edificações verticais: levantamento e transporte de cargas (Seminário de Mestrado). Universidade Federal da Paraíba, João Pessoa, PB, Brasil.

Bankoff, A. D. P., Freire, F. B., \& Villarta, R. (1994). Postura corporal: integração dos fatores culturais e sociais aos fatores biológicos. (1. Ed.). Brasília: Programa de Educação e Saúde através do Exercício Físico e do Esporte - MEC/MS

Borba, J. V. F., \& Soares, B. A. (2013). Avaliação dos riscos ergonômicos do operário na construção civil durante a reforma de uma universidade em Campina Grande. In XXXIII Encontro Nacional de Engenharia de Produção, A Gestão dos Processos de Produção e as Parcerias Globais para o Desenvolvimento Sustentável dos Sistemas Produtivos. Salvador: Autor.

Couto, H. de A. (1991). Guia prático tenossinovites e outras lesões por traumas cumulativos nos membros superiores de origem ocupacional. In Merino, E. A. D. (1996). Efeitos agudos e crônicos causados pelo manuseio e movimentação de cargas no trabalhador (Dissertação de Mestrado). Universidade Federal de Santa Catarina, Florianópolis, SC, Brasil.

Couto, H. de A. (1995). Ergonomia aplicada ao trabalho: manual técnico da máquina humana. In Melo, A. M. (2003). Ergonomia na movimentação de cargas pesadas manual ou com uso de empilhadeiras (Dissertação de PósGraduação). Universidade Cândido Mendes, Rio de Janeiro, RJ, Brasil.

Decreto-Lei $\mathrm{N}^{\circ} 5.452$, de $1^{\circ}$ de maio de 1943. Aprova a Consolidação das Leis do Trabalho. Recuperado de http://www.planalto.gov.br/ccivil_03/ decreto-lei/del5452.htm.

Fundacentro. (1991). Levantamento e transporte manual de pesos. (1. Ed.). São Paulo: Autor.

Grandjean, E. (1982). Fitting the task to the man: an ergonomic approach. (1. Ed.). London: Taylor \& Francis.

Kassada, D. S., Lopes, F. L. P., \& Kassada, D. A. (2011). Ergonomia: atividades que comprometem a saúde do trabalhador. Anais Eletrônico do Encontro Internacional de Produção Científica Cesumar. Centro Universitário de Maringá, Maringá, PR, Brasil, 7.

Medeiros, D. M. (2013). A importância da ergonomia na construção civil: uma revisão. (Especialização). Faculdade Cruzeiro do Sul, Goiânia, GO, Brasil.

Melo, A. M. (2003). Ergonomia na movimentação de cargas pesadas manual ou com uso de empilhadeiras (Dissertação de Pós-Graduação). Universidade Cândido Mendes, Rio de Janeiro, RJ, Brasil.

Mendes, R. (1995). Patologia do trabalho. (1. Ed.) Rio de Janeiro: Atheneu. Mercúrio, R. (1978). O que você deve saber sobre a coluna vertebral. (1. Ed.). São Paulo: Livraria Nobel S.A.

Merino, E. A. D. (1996). Efeitos agudos e crônicos causados pelo manuseio e movimentação de cargas no trabalhador (Dissertação de Mestrado). Universidade Federal de Santa Catarina, Florianópolis, SC, Brasil.

Moura, R. (1978). Segurança na movimentação de materiais. (1. Ed.). São Bernardo do Campo: Ivan Rossi.

Nogueira, D. P. (1987). Prevention of accidents and injuries in Brazil. (2. Ed.). London: Ergonomics.

Nota técnica $\mathrm{n}^{\circ}$ 05/2012/DSST/SIT. Manuseio de sacos de cimento de $50 \mathrm{~kg}$. Recuperado de http://www.abergo.org.br/arquivos/noticias/notatec.pdf.

NR 11, de 08 de junho de 1978. Transporte, movimentação, armazenagem e manuseio de materiais. Recuperado de http://rabalho.gov.br/images/ Documentos/SST/NR/NR11.pdf.

NR 17, de 08 de junho de 1978. Ergonomia. Recuperado de http://trabalho. gov.br/images/Documentos/SST/NR/NR17.pdf.

NR 5, de 08 de junho de 1978. Comissão Interna de Prevenção de Acidentes. Recuperado de http://trabalho.gov.br/images/Documentos/SST/NR/NR5. pdf.

NR 6, de 08 de junho de 1978. Equipamento de Proteção Individual - EPI. Recuperado de https://enit.trabalho.gov.br/portal/images/Arquivos_SST/ SST_NR/NR-06.pdf.

NR 9, de 08 de junho de 1978. Programa de Prevenção de Riscos Ambientais. Recuperado de http://trabalho.gov.br/images/Documentos/SST/NR/NR09/ NR-09-2016.pdf.
Servilha, E. A. M., Leal, R. de O. F., \& Hidaka, M. T. U. (2010). Riscos ocupacionais na legislação trabalhista brasileira: destaque para aqueles relativos à saúde e à voz do professor. Rev. soc. bras. fonoaudiol., 15(4), 1-9. Recuperado de http://www.scielo.br/pdf/rsbf/v15n4/a06v15n4.pdf.

Watters, T. R. Putz-Anderson V, Garg A, \& Fine LJ. (1993). Revised NIOSH equation for the design and evaluation of manual lifting task. In Merino, E. A. D. (1996). Efeitos agudos e crônicos causados pelo manuseio e movimentação de cargas no trabalhador. (Dissertação de Mestrado). Universidade Federal de Santa Catarina, Florianópolis, SC, Brasil. 